\title{
Abdominal Wall Tumors: A Retrospective and Prospective Study.
}

\author{
Alaa El-Din Hassan Mohammed, Asem Elsany Mohammed Ali, Mohammed \\ Yousef Mohammed, Mohammed Abd el All Ali Osman.
}

\section{Abstract}

Background: Abdominal wall masses are not uncommon diseases that manifest with solitary or multiple lesions and can be associated with intraperitoneal diseases, Tumors of the abdominal wall may be benign locally malignant or malignant. This review aims to study the incidence and pattern of abdominal wall tumors in Sohag governorate and to address the different types of these tumors, approach to diagnosis, differential diagnosis, and available treatment options.

Methods: This study was a prospective study which was conducted at the main health institutions in Sohag governorate in the period from January 2000 to April 2017, The study included patients presented with abdominal wall tumors during the period of the study, analysis of the clinical presentation, different methods of diagnosis and treatment varieties.

Results: The study included 25 patients presented with abdominal wall tumor, data were recorded, tabulated and statistically analyzed emphasizing on Preoperative data including presentation and diagnosis, Operative data, intra and post-operative complications, different methods for closure of the defect and early postoperative follow up. 8 cases $(32.63 \%)$ were benign, 1 case $(3.90 \%)$ was locally malignant and 16 cases $(64.67 \%)$ were malignant.

Conclusion: Most of abdominal wall tumors had an idiopathic etiology. CT was the corner stone in diagnosis of abdominal wall tumors and surgical intervention as an option for management of abdominal wall tumors proved its effectiveness and great rule.

\section{Introduction}

The abdominal wall encompasses an area of the body bounded superiorly by the xiphoid process and costal arch, and inferiorly by the inguinal ligament, pubic bones and the iliac crest. It consists of skin, subcutaneous fat, superficial fascia, external and internal abdominal oblique muscles, transversus abdominis muscle, transversalis fascia, properitoneal fat and peritoneum. It protects and contains the abdominal viscera, and serves to position and stabilize the thorax and upper body in space during movement.

Abdominal wall masses are not uncommon diseases that manifest with solitary or multiple, palpable or non palpable lesions and can be associated with intraperitoneal diseases. Tumors of the abdominal wall may be benign such as lipomas, fibromas haemangiomas and endometriomas, locally malignant as desmoid tumour or malignant. In many clinical practices, abdominal wall tumors can be detected by patient's symptoms or physical examination. However unless the typical clinical manifestation, it is difficult to detect or characterize various abdominal masses.

Ultrasonography is easy and convenient method to determine the presence or absence of abdominal wall lesions and it is useful for differential diagnosis with its characteristic imaging findings. Recent development of multi-detector CT technology allowed high resolution body imaging, it can detect more small masses of abdominal wall and it can also evaluate peritoneal pathology. 
After tumor removal, the abdominal wall defects usually cannot be primarily sutured together. Tumor excision of the abdominal wall is limited only by what can be reconstructed. A straightforward analysis of the two related but independent questions of how to repair the internal or structural abdominal wall and how to close the skin leads to a decision tree involving mesh or fascial flap selection and the possible need for a simultaneous skin flap. With proper planning and coordination with the surgical team the reconstruction can be simplified and patient outcomes improved.

\section{Patients and methods}

The study is a prospective study that was conducted at Sohag main health institutions in the period from January 2000 to April 2017 and included patients with any age and sex that were represented with abdominal wall tumors either benign, locally malignant or malignant. Patients with abdominal wall hernias and patients with acute inflammations affecting layers of the abdominal wall were excluded from the study.

All patient demographic data were included. Detailed history and clinical examination were also analyzed. All $\mathrm{lab}$ and imaging investigations were recorded.

Detailed treatment options with special referral to intra-operative data and post operative complications were addressed. The histopathology of the specimens taken also was recorded. Any neo-adjuvant or adjuvant treatment either chemotherapy or radiotherapy were recorded. Follow up of the patient to detect any recurrence was also addressed.

All these statistics were tabulated and analyzed using SPSS program.

Informed consent was taken from all cases. Ethical approval was taken from the scientific ethics committee.

\section{Results}

The study included 25 patients with abdominal wall tumors 14 of them were females $(55.56 \%)$ and 11 were males (44.44\%), Ages of the patients ranged from 2 years to 84 years, benign tumors were found in 8 cases $(32.63 \%)$, while malignant varieties were 16 cases $(64.67 \%)$, locally malignant tumors as desmoid tumor found only in one case $(3.9 \%)$.

From all abdominal wall tumors included in this study, primary tumors arising from different layers of anterior abdominal wall were found in 10 cases (40.44\%), secondary tumors found in 7 cases $(27.93 \%)$, also, extension from intra-abdominal organs found and represented with 8 cases $(32.63 \%)$. Ultrasound was performed in 24 cases $(96.1 \%)$, from all cases sonar was performed, it was diagnostic in 10 cases (40.04\%) of which 5 cases were lipomas, a case of endometrioma, a case of cystic hygroma, and a case of cancer gall bladder extending to the anterior abdominal wall. CT was done in 17 cases $(86.37 \%)$, from cases CT done in them, it was diagnostic in 13 cases $(76.68 \%)$, MRI was performed for diagnosis in 4 cases $(15.81 \%)$, and these cases were a case of lipolymphangima, two cases of cancer bladder extending to anterior abdominal wall, and a case of cancer uterus with anterior abdominal wall metastasis.

After obtaining the biopsy or after the surgery performed for the abdominal wall tumor, biopsy results were sent to tissue laboratories, the results were obtained, recorded and statistically analyzed, sarcomas with different subtypes found in 2 cases $(8.81 \%)$, adenocarcinoma found in 4 cases $(15.81 \%)$, squamous cell carcinoma found in 3 cases $(12.11 \%)$, other types of carcinomas found in 3 cases $(12.11 \%)$, cystic hygroma, endometrioma, lymphoma and spindle cell tumor, all the previous 4 types were found in one case each $(3.9 \%)$, 


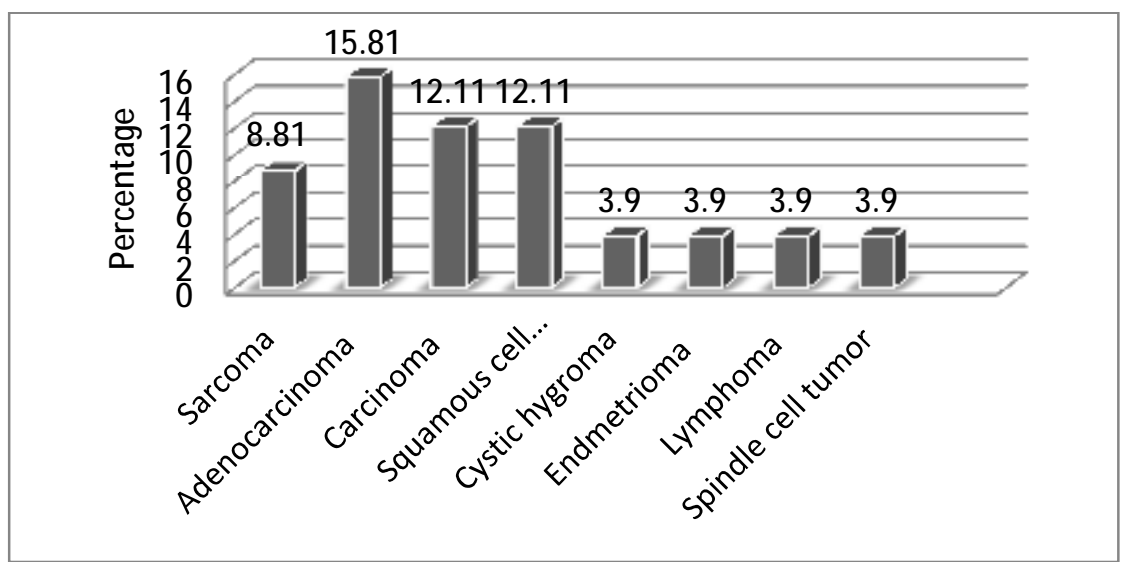

Figure (1): Bar chart showing Distribution of studied population by biopsy results.

Operative management as one of the treatment plans for abdominal wall tumors was performed in 12 cases $(72.67 \%)$. Surgery was followed by adjuvant chemotherapy in 7 cases $(25.0 \%)$, and adjuvant radiotherapy in 2 cases $(8.0 \%)$. Radiotherapy as a method for management of non operable abdominal wall tumors used in 2 cases $(8.0 \%)$. On the other hand, chemotherapy was given in 8 cases $(32.0 \%)$. The tumors that were sensitive to chemotherapy ranged from multiple types as leiomyosarcoma, paraganglioma, sarcomas, rhabdomyosarcoma, B cell lymphoma, adenocarcinomas, and mesothelioma .

From all the previous analyzed data, comparisonal study performed between benign and malignant tumors that predominate in males and in females, females are affected more by benign types of abdominal wall lesions, while in males, malignant varieties are the predominant ones with $\mathrm{P}$ value $=0.24$ with no statistical significance. The mean age for benign lesions is 14.31 years, that explains the young ages of patients presented with benign tumors, but in malignant tumors, the mean age is 56.37 years that shows the old age of presentation with $\mathrm{P}$ value $=0.0003$ which is statistically significant .

Table (1): Imaging modalities and treatment options of the studied patients

\begin{tabular}{|l|l|}
\hline Item & Number of cases \\
\hline Imaging technique & \\
\hline Ultrasound & $24(96.1 \%)$ \\
\hline CT & $17(\mathbf{7 6 . 6 8 \%})$ \\
\hline MRI & $4(15.81 \%)$ \\
\hline Treatment options & \\
\hline Surgery & $\mathbf{1 8}(\mathbf{7 2 . 6 7 \% )}$ \\
\hline Conservative & $5(20.0 \%)$ \\
\hline $\begin{array}{l}\text { Adjuvant } \\
\text { chemotherapy }\end{array}$ & $\mathbf{7 ( 2 5 . 0 \% )}$ \\
\hline Adjuvant radiotherapy & $\mathbf{2 ( 8 . 0 \% )}$ \\
\hline Radiotherapy & $\mathbf{2 ( 8 . 0 \% )}$ \\
\hline Chemotherapy & $\mathbf{8 ( 3 2 . 0 \% )}$ \\
\hline Total number & $\mathbf{2 5 ( 1 0 0 \% )}$ \\
\hline
\end{tabular}


SOHAG MEDICAL JOURNAL

Vol. 21 No.3 october 2017
Abdominal Wall Tumors: A Retrospective and Prospective Study. Mohammed Abd el All Ali

Table (2): Comparison between benign and malignant tumor as regard age and gender

\begin{tabular}{|c|c|c|c|}
\hline Variables & \begin{tabular}{|l|} 
Benign \\
$\mathrm{N}=8$
\end{tabular} & \begin{tabular}{|l|} 
Malignant \\
$\mathrm{N}=16$
\end{tabular} & $P$ value \\
\hline $\begin{array}{l}\text { Age/years } \\
\text { Mean } \pm \text { SD } \\
\text { Median (range) }\end{array}$ & $\begin{array}{l}14.13 \pm 16.39 \\
4.5(2-45)\end{array}$ & \begin{tabular}{|l}
$56.37 \pm 18.84$ \\
$62(14-84)$
\end{tabular} & $\begin{array}{l}0.0003 \\
\text { Statistically significant. }\end{array}$ \\
\hline $\begin{array}{l}\text { Gender } \\
\text { Females } \\
\text { Males }\end{array}$ & $\begin{array}{l}6(75.00 \%) \\
2(25.00 \%)\end{array}$ & $\begin{array}{l}8(47.37 \%) \\
9(52.63 \%)\end{array}$ & $\begin{array}{l}\quad 0.24 \\
\text { With no statistical significance. }\end{array}$ \\
\hline
\end{tabular}

Fig (2): female patient 60 years with umbilical mass (Sister Josef nodules)

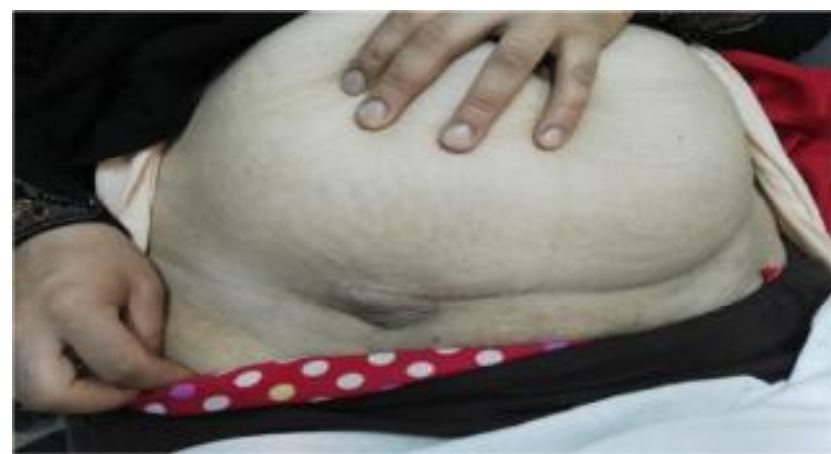

Fig (3): female patient 45 years with endometrioma at site of a previous cesarian section

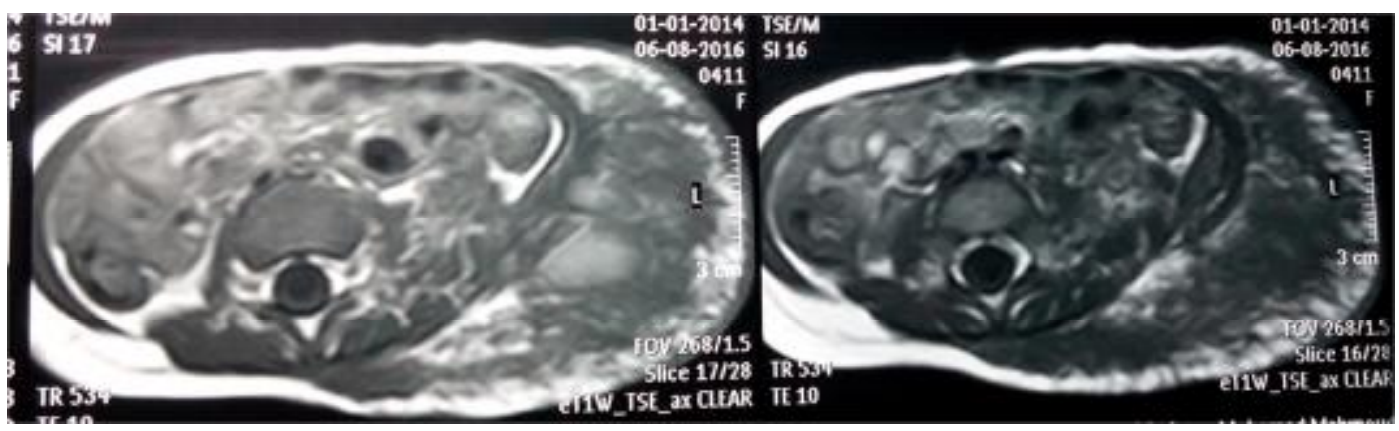

Fig (4): MRI image showing lipolymphangioma at the anterior abdominal wall 


\section{Discussion}

Abdominal wall masses are not uncommon diseases that affect many patients; they may be solitary or multiple swellings, palpable or non palpable lesions at time of examination and may be denovo or can be associated with intraperitoneal diseases. Etiologically; may be inflammatory, post traumatic, or neoplastic with variant types as benign, locally malignant, malignant, or even metastatic.

In our study, we discussed a group of 25 patients with variable types of abdominal wall tumors, age ranged from 2 years to 84 years. abdominal wall tumors affected females more than males with a slight higher percentage especially with benign lesions but malignant lesions were found more in males (Arslan, 2005).

To identify the mass seen or felt via abdominal examination, radiological investigations had to be done, Ultrasonography was an easy method to determine the presence or absence of abdominal wall lesions and was the modality of choice in the routine work up of any abdominal wall mass and it was useful for differential diagnosis between different types of abdominal wall masses with its characteristic imaging findings (Lee et al, 2009).

Recent development of multi-detector CT technology allowed high resolution body imaging, it detected smaller masses of abdominal wall and it allowed to differentiate multiple masses from each other and could also evaluate peritoneal pathology. Also it was very effective in determining the dimensions of the abdominal wall tumor (Ademuyiwa et al, 2010).

In this work, CT was the rule for diagnosis in 13 cases $(76.68 \%)$ as sonar was not diagnostic in these cases. These cases had mainly malignant abdominal wall tumors.
The advantages and the upper hand of MRI over CT and sonar were obvious and used if sonar and CT weren't able to differentiate the abdominal wall mass but it had also the drawbacks of being very costly to the patient and exposure to higher doses of radiation. MRI was done only for two cases in our study.

From all abdominal wall tumors included in our study, primary tumors that are arising from the different layers of abdominal wall were $40.44 \%$, secondary tumors that involve the abdominal wall were $27.93 \%$, extension of intra-abdominal tumors was also represented and found in about $32.63 \%$ of cases this was comparable with results of saxe et al, 2003.

Lipomas of the anterior abdominal wall were the most commonly occurring tumor of the abdominal wall with incidence of 5 cases of 25 cases $(20.0 \%)$ near the incidence of Murphey et al, 2014.

Endometrioma as one of benign abdominal wall lesions found mainly at site of scars of C.S.; in our study, the incidence of endometriomas is 1 of 25 cases $(4.0 \%)$ roughly the same as Erkan et al, 2004 which included 400 cases of whom 16 cases were endometriomas.

Abdominal wall sarcomas with its different types were discussed in our study by 2 of 25 cases $(8.81 \%$ ) a slightly higher incidence than Storm, 2008.

Surgical intervention as a method for management of abdominal wall tumors was performed in $72.6 \%$ of cases, more in malignant tumors $(73.6 \%)$ of malignant tumors included in our study.

Chemotherapy constitutes a cornerstone modality that 8 of 25 cases $(32.0 \%)$ received it as the main stay of treatment of non operable malignant 
abdominal wall tumor (Biluts and Yoo, 2007).

Radiotherapy also constituted a non negligible modality in management of abdominal wall tumors depending on the histopathological type of the malignant tumor and in our study about $8 \%$ of cases of malignant masses received radiotherapy

(Dumanian, 2009).

\section{Conclusion}

Most of abdominal wall tumors had an idiopathic etiology, but may be related to chronic irritation and exposure to radiations or neglected hematoma post traumatic.

CT was the corner stone in diagnosis of abdominal wall tumors that allowed high resolution body imaging\& its ability to detect more small masses of abdominal wall and it differentiates multiple masses from each other and can also evaluate peritoneal pathology. Surgical intervention as an option for management of abdominal wall tumors had proved its effectiveness and great rule.

\section{Ethical Considerations}

Informed consent was taken from all cases. Ethical approval was taken from the scientific ethics committee.

\section{Conflict of Interest}

The authors declare that they don't have any conflict of interest.

\section{References}

1. Arslan OE. Anatomy of the abdominal wall, In Shiffman MA, Mirrafati S (Eds.). Aesthetic surgery of the abdominal wall, 2005, Springer, Berlin, Heidelberg, Germany.

2. Marley MH. Radiation therapy for aggressive fibromatosis (desmoid tumors): results of a national Patterns of Care Study. Int J Radiat Oncol Biol Phys 2005; 61: 882-91.

3. Lee JC, Thomas JM, Phillips S. Aggressive fibromatosis: MRI features with pathologic correlation. Am J Roentgenol 2006; 186: 247-54.

4. Wong SL. Diagnosis and management of desmoid tumors and fibrosarcoma. $\mathrm{J}$ Surg Onc 2008; 97: 554-8.

5. Ademuyiwa AO, Bode CO, Elebute OA. Anterior abdominal wall desmoid tumor in a five year old girl - a pre operative diagnostic challenge in resource-poor setting. Ann Ped Surg 2010; 6: 41-3.

6. Saxe JM, Ledgerwood AM, Lucas CE. Management of the difficult abdominal closure. Surg Clin NorthAm 2003; 73 (2): 243-51.

7. Erkan N, Haciyanli M, Sayhan H. Abdominal wall endometriomas. Int $\mathbf{J}$ Gyne Obstet 2005; 89: 59-60.

8. Storm HH. Survival of adult patients with cancer of soft tissues or bone in Europe. Eur J Cancer 2008; 34(14): 2212-7.

9. Biluts H, Yoo M. Abdominal wall tumours in Tikur Anbessa Hospital in Addis Ababa, Ethiopia .Association of surgeons of East Africa and College of Surgeons of East Central and Southern Africa, 2007, surgery @ telecom.net.et.

10. Dumanian GA. Abdominal wall tumors and their reconstruction. In Butler CE and Fine NA (eds.). Principles of cancer reconstructive surgery, 2008, Springer-Verlag Berlin Heidelberg, Germany. 